\title{
Use of a Patient-Friendly Terms List in the Adverse Drug Reaction Report Form: A Database Study
}

\author{
Sieta T. de Vries ${ }^{1}$ (D) Judy Harrison ${ }^{2} \cdot$ Patrick Revelle $^{2} \cdot$ Alicia Ptaszynska-Neophytou $^{3} \cdot$ Anna Radecka $^{3}$. \\ Gowthamei Ragunathan ${ }^{3} \cdot$ Phil Tregunno ${ }^{3} \cdot$ Petra Denig $^{1}$ (D) Peter G. M. Mol ${ }^{1,4}$ (I)
}

Published online: 6 February 2019

(c) The Author(s) 2019

\begin{abstract}
Introduction When reporting adverse drug reactions to pharmacovigilance centres, patients and consumers can describe adverse drug reactions experienced in free-text format. Recently, a patient-friendly adverse drug reaction terms list was introduced in the adverse drug reaction report form in the UK to facilitate this reporting.

Objective The objective of this study was to evaluate the actual use of the patient-friendly terms list in the adverse drug reaction report form and its association with the type of adverse drug reactions reported.

Methods We conducted a database study in which we reviewed the list's use for all reported adverse drug reactions by patients and consumers to the pharmacovigilance centre in the UK via the online report form between August and September 2017. Descriptive statistics were used. In addition, for adverse drug reactions reported more than 20 times, Chi-squared tests were used to test for differences in the number of reports in which the patient-friendly terms list was used and those in which the adverse drug reaction was entered as free text.

Results In total, 888 reports were received. In 185 reports (21\%), the patient-friendly terms list was used to enter an adverse drug reaction. In total, the reports contained 3227 adverse drug reactions. Nausea, headache, diarrhoea, dizziness, insomnia, anxiety, depression, fatigue, tiredness, vomiting, appetite lost, joint pain, chest pain, constipation and pain were reported more than 20 times. Five of these adverse drug reactions (i.e. nausea, diarrhoea, dizziness, insomnia and constipation) were reported significantly more often in reports where the adverse drug reactions were selected from the patient-friendly terms list.

Conclusions Most people chose to describe adverse drug reactions in their own words rather than selecting adverse drug reactions from a patient-friendly terms list. Although the patient-friendly terms list may be a useful feature for some patients or for some adverse drug reactions, it should not replace the option for patients to describe adverse drug reactions in their own words.
\end{abstract}

\section{Key Points}

Electronic supplementary material The online version of this article (https://doi.org/10.1007/s40264-019-00800-x) contains supplementary material, which is available to authorized users.

\section{Peter G. M. Mol}

p.g.m.mol@umcg.nl

1 Department of Clinical Pharmacy and Pharmacology, University of Groningen, University Medical Center Groningen, Hanzeplein 1, PO Box 30001, 9700 Groningen, RB, The Netherlands

2 MedDRA® Maintenance and Support Services Organization, McLean, VA, USA

3 Medicines and Healthcare products Regulatory Agency, London, UK

4 Dutch Medicines Evaluation Board, Utrecht, The Netherlands
People reporting adverse drug reactions to a pharmacovigilance centre more often describe their experiences using free text rather than by selecting a term from a list.

The use of a patient-friendly terms list may depend on the type of adverse drug reaction reported.

The patient-friendly terms list may support some reporters but should not replace the possibility for people to describe an adverse drug reaction in their own words. 


\section{Introduction}

Post-marketing surveillance is crucial for monitoring a drug's safety profile after its market approval. In the past, reporting of adverse drug reactions (ADRs) to the national agency could only be completed by healthcare professionals. Over time, the interest in reports submitted by patients or consumers has increased, as they are seen as an important source of information about ADRs [1-3]. Currently, many pharmacovigilance centres worldwide allow both patients and consumers and healthcare professionals to report ADRs directly to the national agency [4].

There are differences across pharmacovigilance centres in the ADR reporting process, for instance, in the manner in which people can report ADRs (e.g. via telephone, paper forms, web-based forms, mobile application), the criteria within the report form and how the reporter can answer the questions in the report form (e.g. free text, radio button, drop-down lists) [5-7]. In most report forms, individuals can describe the ADR experienced as free text. In the online report form of the Medicines and Healthcare products Regulatory Agency (MHRA) in the UK (i.e. the Yellow Card scheme) [8], individuals can also select ADR terms from a list that appears after a few letters are entered in addition to having a free-text field to describe their experience. The list is made up of terms from the Medical Dictionary for Regulatory Activities (MedDRA®) [9]—a standardised international terminology-and selected terms can be directly transferred into the MHRA database without the need for manual coding from free text.

The use of a list to select ADRs in an online report form could thus reduce the time needed for assessors to code reported ADRs and could ease the process of reporting an ADR. However, in a small pilot study that we conducted, patients indicated they preferred to describe an ADR experienced in their own words rather than selecting a term from a list. Reasons for this preference were that they thought more accurate information could be provided using their own words when using free text and that they were concerned with the difficulty of understanding medical terms used in such a list [see Electronic Supplementary Material 1]. The latter might be resolved by using a list with patient-friendly terms. Such patient-friendly terms have been proposed for reporting tools used by patients [10]. Additionally, a qualitative study showed that the use of patient-friendly terms is an important factor for using a mobile application for ADR reporting [11]. However, for some ADRs, patients may still prefer to use their own words.

Recently, a "patient-friendly list" of MedDRA® was developed in English by the MedDRA ${ }^{\circledR}$ Maintenance and Support Services Organization and other partners of the
Web-Recognising Adverse Drug Reactions (WEB-RADR) project [12]. This list includes patient-friendly terms for approximately 1400 of the lowest level terms (LLTs) from MedDRA ${ }^{\circledR}$ and is available online [13]. It contains ADR terms, using patients' and consumers' own texts, of the most frequently reported ADRs by individuals to pharmacovigilance centres. The patient-friendly terms list thus generally reflects the language used by individuals submitting ADR reports but is not (yet) comprehensive. Some of the terms on the list are of a technical or medical nature and might not represent the typical language some individuals would use to report that ADR. It is possible that, whilst the ADR was originally reported by a patient, another individual may have assisted and translated the ADR into more medical language for the purpose of submitting the report. Therefore, some terms on the list may still be difficult for people to understand (see the ESM 1).

In this study, we aimed to evaluate the usefulness of the currently available patient-friendly terms list for reporting ADRs by patients and consumers. More specifically, our aims were to evaluate the actual use of the patient-friendly terms list and its association with the type of ADRs reported.

\section{Methods}

We conducted a database study in the context of the WEBRADR project. Data of ADRs spontaneously reported by patients and consumers through the MHRA's Yellow Card scheme online form were used.

\subsection{Procedure and Included Reports}

The patient-friendly terms list developed by the MedDRA® Maintenance and Support Services Organization and other partners of the WEB-RADR project was incorporated in the patient and consumer version of the Yellow Card scheme online form (https://yellowcard.mhra.gov.uk/) on 2 August, 2017. The patient-friendly terms appear when a person enters a few letters in the text field to describe the ADR. The ADR terms that appear contain the exact entered letters; they do not include other medically related MedDRA $®$ terms that do not contain the entered letters. For an example, see the ESM 2. ADRs selected from the patient-friendly terms list were recorded as MedDRA ${ }^{\circledR}$ LLTs in the database, whereas ADRs entered as free text were linked to MedDRA® LLTs by trained pharmacovigilance assessors.

The data for this study were extracted on 18 September, 2017. All reports received in this time period from patients and consumers were included. We assessed how many people selected an ADR from the list or entered the ADR as free text, and which MedDRA ${ }^{\circledR}$ terms were documented via both methods of ADR reporting. Use of the patient-friendly terms 
list was assumed when there was an exact match between the reported ADR and a term from the list, considering that the drop-down list appears when typing the first letters of a term on the list.

\subsection{Analyses}

Descriptive statistics were used to describe the number of received reports per reporting method, the number of ADRs at the LLT level, and the sex and age of the patients for which an ADR was reported. The association between the reporting methods and the type of reported ADRs was assessed for the ADRs of which more than 20 reports were received. Chi-squared tests or Fisher's exact tests in the case of cells with fewer than five cases were used to test for differences between the reporting methods in the number of reports received for these ADRs. $P$-values $<0.05$ were considered statistically significant. The analyses were conducted using Stata Version 14.2 (Stata Corp., College Station, TX, USA).

\section{Results}

There were 888 ADR reports received in the study period. In $69 \%$ of the reports, a female patient was involved. The age ranged from 1 to 94 years (mean: 45, standard deviation: 20). In 185 reports (21\%), the patient-friendly terms list was used to enter the ADR(s). In 137 reports (15\%), some ADRs were reported as free text while other ADRs were selected from the patient-friendly terms list. Reports in which the list was used concerned younger patients than the reports in which the ADRs were entered as free text (on average 37 years vs. 49 years; Table 1 ).

In total, the reports contained 3227 ADRs of which nausea, headache, diarrhoea, dizziness, insomnia, anxiety, depression, fatigue, tiredness, vomiting, appetite lost, joint pain, chest pain, constipation and pain were reported more than 20 times. Five of these ADRs (i.e. nausea, diarrhoea, dizziness, insomnia and constipation) were more often mentioned in reports where the ADR was selected from the patient-friendly terms list (Table 2). Pain was more often mentioned using free text $(21$ reports vs. 0 reports; $p=$ 0.017). Depression was also only mentioned in reports using free text. However, this term was not included in the patientfriendly terms list. An overview of all reported ADRs at the LLT level is presented online (see the ESM 3).

\section{Discussion}

Our study shows that for the majority of the reports to a pharmacovigilance centre, people chose to describe the ADRs in their own words rather than selecting ADRs from a patient-friendly terms list. Still, one fifth of the people used the patient-friendly terms list when reporting an ADR. These patients were on average more than 10 years younger. It seems that the use of the patient-friendly terms list depends on the ADR it concerns.

Our results indicate that from a patient perspective, the free-text option to describe an ADR should be available and should not be replaced by a list for selecting ADRs. Free text provides individuals the option to use their own words. Previously, it has been shown that patients provide richer and more detailed information about ADRs in a report than healthcare professionals [14]. This suggests that information could be lost when people cannot describe the ADRs they experienced in their own words. A recent study also showed that this additional information provided by patients is important in the identification and assessment of safety signals [15]. The MHRA currently allows a hybrid approach where terms are presented in a drop-down list, but do not necessarily have to be selected. Additionally, there is a large free-text field, which allows for unstructured data and different patient preferences.

The use of a patient-friendly terms list in the ADR report form may have advantages for the handling of the reports. For instance, it is expected to save time when assessors do not need to code a description of an ADR as entered by patients and consumers to the terms provided in MedDRA®. However, guidelines about MedDRA® coding already mention the challenge of selecting the right term for

Table 1 Characteristics of the adverse drug reaction (ADR) reports

\begin{tabular}{llllr}
\hline & Overall & $\begin{array}{l}\text { Reports in which ADRs were } \\
\text { entered as free text }\end{array}$ & $\begin{array}{l}\text { Reports in which ADRs were selected } \\
\text { from the patient-friendly terms list }\end{array}$ & Use of both options \\
\hline Number of reports $(\%)$ & 888 & $566(64)$ & $185(21)$ & $137(15)$ \\
Female sex $(\%)^{\mathrm{a}}$ & $613(69)$ & $372(66)$ & $140(76)$ & $101(74)$ \\
Age, mean (SD) & $45(20)$ & $49(20)$ & $37(16)$ & $40(18)$ \\
\hline
\end{tabular}

$S D$ standard deviation

${ }^{\text {a }}$ Sex was unknown in 4 reports

${ }^{\mathrm{b}}$ Age was missing in 64 reports 
Table 2 Characteristics of the adverse drug reactions (ADRs) entered as free text and selected from the patient-friendly terms list

\begin{tabular}{|c|c|c|c|c|}
\hline & Overall & $\begin{array}{l}\text { Reports in which ADRs } \\
\text { were entered as free text }\end{array}$ & $\begin{array}{l}\text { Reports in which ADRs were } \\
\text { selected from the patient-friendly } \\
\text { terms list }\end{array}$ & $P$ value $^{\mathrm{a}}$ \\
\hline $\begin{array}{r}\text { Number of ADRs } \\
\text { at the LLT level }\end{array}$ & 3227 & 2545 & 682 & \\
\hline \multicolumn{5}{|c|}{ Overall, 10 most commonly reported ADRs (\% of number of ADRs at the LLT level) } \\
\hline Nausea & $78(2.4)$ & $54(2.1)$ & $24(3.5)$ & $\mathbf{0 . 0 3 5}$ \\
\hline Headache & $77(2.4)$ & $65(2.6)$ & $12(1.8)$ & 0.227 \\
\hline Diarrhoea & $62(1.9)$ & $36(1.4)$ & $26(3.8)$ & $<0.001$ \\
\hline Dizziness & $54(1.7)$ & $36(1.4)$ & $18(2.6)$ & 0.027 \\
\hline Insomnia & $33(1.0)$ & $20(0.8)$ & 13 (1.9) & 0.010 \\
\hline Anxiety & $29(0.9)$ & $21(0.8)$ & $8(1.2)$ & 0.393 \\
\hline Depression & $28(0.9)$ & $28(1.1)$ & $0(0.0)^{\mathrm{b}}$ & $0.002^{\mathrm{c}}$ \\
\hline Fatigue & $28(0.9)$ & $23(0.9)$ & $5(0.7)$ & 0.670 \\
\hline Tiredness & $28(0.9)$ & $22(0.9)$ & $6(0.9)$ & 0.969 \\
\hline Vomiting & $28(0.9)$ & $19(0.8)$ & $9(1.3)$ & 0.152 \\
\hline Appetite lost & $26(0.8)$ & $21(0.8)$ & $5(0.7)$ & 0.811 \\
\hline Joint pain & $24(0.7)$ & $18(0.7)$ & $6(0.9)$ & 0.641 \\
\hline Chest pain & $23(0.7)$ & $18(0.7)$ & $5(0.7)$ & 0.943 \\
\hline Constipation & $21(0.7)$ & $11(0.4)$ & $10(1.5)$ & 0.003 \\
\hline Pain & $21(0.7)$ & $21(0.8)$ & $0(0)$ & $0.013^{\mathrm{c}}$ \\
\hline
\end{tabular}

$L L T$ lowest level term

${ }^{a}$ Measured using Chi-squared tests

${ }^{\mathrm{b}}$ This ADR was not on the patient-friendly terms list

${ }^{c}$ Measured using the Fisher's exact test professionals, where for instance a difference of one letter can impact the meaning of a symptom [16]. It is questionable whether patients and consumers will be able to make such distinctions when selecting terms from a predefined list. It is therefore important that the terms in the list are clear and unambiguous. Further studies are needed to assess the validity of reports in which ADRs are selected from a patient-friendly terms list. In particular, it should be assessed whether the terms sufficiently and correctly reflect what the patients experienced.

Our study showed that the type of ADR may influence the use of the patient-friendly terms list. It could be that for some ADRs the term is so unambiguous that more people are content to select it from a list, as we observed for nausea, diarrhoea, dizziness, insomnia and constipation. Moreover, a less specific ADR, i.e. pain, was shown to be more often reported using free text. However, other ADRs that seem unambiguous, such as headache or fatigue, were not more often selected from the list. More in-depth studies are needed to understand when individuals prefer to describe ADRs in their own words. Furthermore, we observed that reports of ADRs using the patient-friendly terms list concerned patients that were generally younger than when free text was used. We do not know whether older people had difficulties with the terms in the list or had a preference for entering the ADR as free text. A previous study showed that older people may have more trouble with drop-down lists [17]. Furthermore, older people may also experience different types of ADRs [18], which may be more difficult to select from a list. Although the person for whom an ADR is reported is not necessarily the same person as the individual sending the report (e.g. a daughter/son reporting an ADR for an elderly parent), our results suggest that more research is needed to assess the association between patient characteristics and the preference or ability to select ADRs from a patient-friendly terms list.

For more widespread use in regulatory and industry online ADR reporting portals, the patient-friendly terms list will need to evolve with the deletion of terms that are difficult to understand and the addition of terms that are more appropriate for use by patients and consumers. To date, the list has only been developed in English; however, MedDRA ${ }^{\circledR}$ is available in ten other languages, including French, Spanish, Dutch, Japanese and Chinese. The original list was derived from actual patient reports in English. Therefore, for other languages, data sources of patient-reported terms are needed to develop appropriate lists specific to those languages. It may not be appropriate to take the English list and convert it into other languages because a term that is a frequently reported and well-understood term in English may not necessarily be a "patient-friendly" or common colloquial expression in another language. 


\subsection{Strengths and Limitations}

This study is the first in which the use of the patient-friendly terms list of MedDRA ${ }^{\circledR}$ in an ADR report form was evaluated. A limitation is that we assumed an exact match between the reported ADR and the ADR on the patientfriendly terms list as an indication of actual use of the list, while a person could still have fully typed the ADR that popped up from the list. Another limitation is that the list does not yet cover all ADRs and that there may not be a term best suited to describe the ADR. Finally, the duration of data collection, i.e. 1.5 months, may seem limited. However, 888 reports were received in this period, which is in our opinion sufficient for an explorative assessment of the use of the patient-friendly terms list.

\section{Conclusion}

This study shows that most individuals choose to describe the ADRs in their own words rather than selecting ADRs from a patient-friendly terms list when they report ADRs to a pharmacovigilance centre. Therefore, the patient-friendly terms list may be a useful feature for some patients or for some ADRs but it should not replace the option for individuals to describe an ADR in their own words. Future studies are needed to understand which people and which ADRs the patient-friendly terms list may be a useful feature for an ADR report form.

Acknowledgements The authors acknowledge Mick Foy, Sarah Vaughan, Rebecca Owen and Bhavini Vyas for their contribution to the data extraction and validation.

Author Contributions All authors contributed to the development and formulation of the research question. APN, AR, GR and PT were involved in the data extraction. STdV conducted the analyses. All authors contributed to the interpretation of the data. STdV wrote the manuscript. JH, PR, APN, AR, GR, PT, PD and PGMM reviewed and edited the manuscript. All authors have read and approved the final manuscript.

\section{Compliance with Ethical Standards}

Funding This study was conducted in the context of the WEB-Recognising Adverse Drug Reactions (WEB-RADR) project. The WEBRADR project has received support from the Innovative Medicines Initiative Joint Undertaking (www.imi.europa.eu) under Grant Agreement No. 115632, the resources of which comprise a financial contribution from the European Union's Seventh Framework Programme FP7/2007-2013 and the EFPIA companies' in kind contribution (www. imi.europa.eu, UK).

Conflict of Interest Sieta T. de Vries, Petra Denig and Peter G.M. Mol have no conflicts of interest that are directly relevant to the content of this study. Alicia Ptaszynska-Neophytou, Anna Radecka, Gowthamei
Ragunathan and Phil Tregunno are employees of the Medicines and Healthcare products Regulatory Agency (MHRA). The research leading to these results was conducted as part of the WEB-RADR consortium (http://webradr.eu), which is a public-private partnership coordinated by the MHRA. Judy Harrison is a consultant and Patrick Revelle is an employee of the MedDRA® Maintenance and Support Services Organization through its parent company, Northrop Grumman. The Maintenance and Support Services Organization maintains and distributes MedDRA®, a standardised international medical terminology. MedDRA $®$ is available by a sliding-scale subscription model for commercial organisations but is provided free to regulatory authorities, non-profit organisations, direct patient care providers and educational institutions. The patient-friendly terms list referenced in the manuscript is freely and publicly available on the MedDRA ${ }^{\circledR}$ website.

Ethics Approval Formal ethical evaluation was not considered necessary for the study described in this report because the data were collected using the results from the MHRA's structural work activities.

Open Access This article is distributed under the terms of the Creative Commons Attribution-NonCommercial 4.0 International License (http://creativecommons.org/licenses/by-nc/4.0/), which permits any noncommercial use, distribution, and reproduction in any medium, provided you give appropriate credit to the original author(s) and the source, provide a link to the Creative Commons license, and indicate if changes were made.

\section{References}

1. de Langen J, van Hunsel F, Passier A, de Jong-van den Berg L, van Grootheest K. Adverse drug reaction reporting by patients in the Netherlands: three years of experience. Drug Saf. 2008;31(6):515-24.

2. Inacio $P$, Cavaco A, Airaksinen M. The value of patient reporting to the pharmacovigilance system: a systematic review. Br J Clin Pharmacol. 2017;83(2):227-46.

3. Blenkinsopp A, Wilkie P, Wang M, Routledge PA. Patient reporting of suspected adverse drug reactions: a review of published literature and international experience. Br J Clin Pharmacol. 2007;63(2):148-56.

4. Matos C, Harmark L, van Hunsel F. Patient reporting of adverse drug reactions: an international survey of national competent authorities' views and needs. Drug Saf. 2016;39(11):1105-16.

5. van Hunsel F, Harmark L, Pal S, Olsson S, van Grootheest K. Experiences with adverse drug reaction reporting by patients: an 11-country survey. Drug Saf. 2012;35(1):45-60.

6. Bailey C, Peddie D, Wickham ME, Badke K, Small SS, DoyleWaters MM, et al. Adverse drug event reporting systems: a systematic review. Br J Clin Pharmacol. 2016;82(1):17-29.

7. Bandekar MS, Anwikar SR, Kshirsagar NA. Quality check of spontaneous adverse drug reaction reporting forms of different countries. Pharmacoepidemiol Drug Saf. 2010;19(11):1181-5.

8. MHRA. Yellow Card scheme online reporting form. https://yello wcard.mhra.gov.uk/. Accessed 18 Dec 2018.

9. MedDRA ${ }^{\circledR}$. Medical dictionary for regulatory activities. https:// www.meddra.org/. Accessed 18 Dec 2018.

10. Banerjee AK, Okun S, Edwards IR, Wicks P, Smith MY, Mayall SJ, et al. Patient-reported outcome measures in safety event reporting: PROSPER consortium guidance. Drug Saf. 2013;36(12):1129-49.

11. de Vries ST, Wong L, Sutcliffe A, Houyez F, Ruiz CL, Mol PG, et al. Factors influencing the use of a mobile app for reporting 
adverse drug reactions and receiving safety information: a qualitative study. Drug Saf. 2017;40(5):443-55.

12. WEB-RADR. WEB-RADR project. https://web-radr.eu/. Accessed 18 Dec 2018.

13. MedDRA ${ }^{\circledR}$. Patient-friendly terms list. https://www.meddra.org/ patient-friendly-term-list. Accessed 18 Dec 2018.

14. Avery AJ, Anderson C, Bond CM, Fortnum H, Gifford A, Hannaford PC, et al. Evaluation of patient reporting of adverse drug reactions to the UK 'Yellow Card Scheme': literature review, descriptive and qualitative analyses, and questionnaire surveys. Health Technol Assess. 2011;15(20):1-234, iii-iv.

15. Watson S, Chandler RE, Taavola H, Härmark L, Grundmark B, Zekarias A, et al. Safety concerns reported by patients identified in a collaborative signal detection workshop using VigiBase: results and reflections from Lareb and Uppsala Monitoring Centre. Drug Saf. 2018;41(2):203-12.

16. MedDRA ${ }^{\circledR}$. MedDRA ${ }^{\circledR}$ term selection: points to consider document. www.meddra.org/how-to-use/support-documentation. Accessed 18 Dec 2018.

17. Dickinson A, Eisma R, Gregor P. The barriers that older novices encounter to computer use. Univ Access Inf Soc. 2011;10(3):261-6.

18. Yu YM, Shin WG, Lee JY, Choi SA, Jo YH, Youn SJ, et al. Patterns of adverse drug reactions in different age groups: analysis of spontaneous reports by community pharmacists. PLoS One. 2015;10(7):e0132916. 